Wick requar of

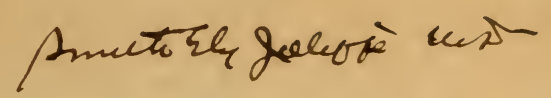




\section{THE PLANTS OF PROSPECT PARK.}

By SMith ELY JELlifFe, M. D., Curator of Herbartum of the Brooklyn Institute.

Phanerogamia-Flowering Plants.

EXOGENS.

Clematis Virginiana.

Anemone nemorosa.

Hepatica.

Anemonella thalictroides.

Aquilegia caerulea.

Ranunculus fascicularis.

$\begin{array}{ll}" 6 & \text { abortivus. } \\ " & \text { bulbosus. } \\ \cdots & \text { acris. } \\ \text { recurvatus. }\end{array}$

Magnolia glauca.

$$
\text { " tripetala. }
$$

Liriodendron Tulipifera.

Berberis vulgaris.

" Mahonia.

Akebia quinata.

Chelidonium majus.

Sanguinaria Canadensis.

Sisymbrium officinale.

Draba verna.

Capsella bursapastoris.

Barbarea vulgaris.

Viola lanceolata.

" palmata, var. cucullata.

" sagittata.

". pubescens.

" tricolor

Tamarisk Gallica.

Cerastium viscosum. arvense, var. oblongifolium.

Scleranthus annuus.

Stellaria, sp.

Claytonia Virginica.

Tilia Americana.

" heterophylla.

“ Europæa.

Geranium maculatum.

Impatiens biflora.

Oxalis violacea. stricta.

Ilex opaca.

Ptelea trifoliata.

Ailanthus glandulosus.

Ampelopsis quinquefolia.

Acer rubrum.

" dasycarpum

" Saccharinuul.

" campestris.

“ pseudo platanus.

“. platanoides.

" circinatum.

" polymorphum.

" macrophyllum.

Negundo aceroides.

Rhus glabra

". Toxicodendron. copallina.

Asculus Hippocastanum. flava.

$$
\text { " Pavia. }
$$

Trifolium pratense.

$$
\begin{array}{ll}
\text { " } & \text { propens. } \\
\text { " hybridumens. }
\end{array}
$$

Melilotus alba.

Wistaria frutescens.

$$
\text { Sinensis. }
$$

Robinia pseud acacia.

Caragana arborescens.

Colutea arborescens.

lophara Japonica.

Ainorpha fruticosa.

Vicia sativa.

Amphicarpea monoica.

Cercis Canadensis.

Gleditschia triacanthus.

Cladrastis tinctoria.
Gymnocladus dioicus.

Prunus Virginiana.

$$
\text { " nana. }
$$

" serotina.

Pyrus arbutifolia.

" ducuparia.

" malus.

" Americana.

Spiræa chamædrifolia, var. simplex.

" hypercifolia.

Amelanchier Canadensis.

Potentilla Canadensis.

Geum vernum.

Cydonia Japonica.

Fragaria Virginiana.

Rubus strigosus.

" odoratus.

Cratægus Oxycantha.

$$
\text { “ } \quad \text { coccinia. }
$$

Calycanthus floridus.

Ribes aureum.

Sdxifraga Virginiensis.

Tiarella cordifolia.

Itea Virginica.

Philadelphus coronarius.

Deutzia gracilis.

Sicyos angulatus.

Hamamelis Virginica.

Liquidamber Stryaciflua.

Fothergilla alnifolia.

Fnothera biennis.

Circæa Lutetiana.

Daucus Carota.

Thaspium aureum.

"var cordatum

Hydrocotyle Americanum.

Osmorrhiza longistylis.

Cryptotænia Canadensis.

Aralia spinosa.

" racemosa.

Cornus florida.

$$
\text { “" alterififolia. }
$$

Lonicera Tartarica.

Diervilla Japonica.

Viburnum Lantana. opulus.

Symphoricarpus racemosus. vulgaris.

Sambucus Canadensis.

Eupatorium ageratoides.

Aster corymbosus.

" sagittifolias.

" patens.

" cordifolius.

"6 " var.laevigatus

" diffusus.

"Tradescanti.

Ambrosia artemisiæfolia.

" trifida.

Xanthium spinosum. strumarium.

Solidago rugosa.

“ neilorosa.

" cessia.

" bicolor.

" Cauadensis.

" lanceolata.

Helianthus annus.

Antlemis Cotula.

Achillea millefolium.

Galinsoga parviflora.

Antennaria plantaginifolia.

Rudbeckia hirta.

Bidens cernua.
Chrysanthemum Leucanthemum.

Cichorium Intybus.

Erigeron Canadensis.

Lappa, Arctium. officinalis.

Taraxacum officinalis.

Vaccinium corymbosum.

Azalea Indica.

" Amæna.

Rhodadendron nudiflorum.

$$
\text { "6 } \quad \text { maximum. }
$$

Andromeda floribunda.

Clethra alnifolia.

Kalmia latifolia.

Stachyuris præcox.

Rbodora Canadensis.

Halesia tetraptera.

Fraxinus Americanus. ornus.

Paulownia imperialis.

Olea Europeæ.

Syringa vulgaris.

"" Persica.

Forsythia viridissima. suspensa.

Chionanthus Americana.

Ligustrum vulgare.

Vinca minor.

Aphyllon uniflorum.

Linaria vulgaris.

Cbelone glabra.

Pedicularis Canadensis.

Verbascum Thapsus.

Veronica peregrina. agrestis.

Tecoma radicans

Catalpa bignonoides.

Mentha Canadensis.

Brunella vulgaris.

Nepeta Cataria. Glechoms.

Lycium vulgare.

Plantago major. lanceolata.

Phytollacea decandra.

Polygonum aviculare.

" dumetorum.

" sagittatum.

Rumex acetosella. Persicaria.

Sassafras officinale.

Ulmus Americana. campestris.

Euphorbia Cyparissias.

Morus alba.

Celtis occidentalis.

Platanus oceidentalis.

Juglans nigra.

Hicoreus albus. por'cina.

Quercus palustris.

Castauea vesea.

Fagus ferruginea.

" pendulis. sylvatica purpurea.

Ostrya Virginica.

Carpinus Americanus.

Betula lenta.

" alba.

" nimra.

Alnus serrulata.

Salix cordata.

" Babylonica.

Populus monolifera.

" heterophylla

" argentea.

.. balsamifera.

dilatata. frondosa. 


\section{THE PLANTS OF PROSPECT PARK-Continued.}

Iris versicolor.

Sisyrinchium angustifolium. Narcissus poeticus.

Smilax glauca

Muscari botryoides.

Convallaria majalis.

Allium Canadensis.

$$
\text { vineale. }
$$

Polygonatum biflora.

Unifolium racemosa. bifolium.

Ornithogalum umbellatum.

Erythronium Americanum.

Scilla sp.

Uvularia perfoliata.

Oakesia sessifolia,

Crocus vernus.

Commelina Virginica.

Typha latifolia.

Arisaema triphyllum.

Luzula campestris.

Carex vulpinoidea.

.. laxiflora

" canescens

“ Pennsylvanica.

“ tentaculata.

Poa annua

Phleum pratense.

Authoxanthum odoratum.

Setaria glauca.

Panicum crus-galli.

Cynodon Dactylon.

Thuja occidentalis.

Juniperus communis.

Taxus baccata.
Hypoxys erecta.

" Canadense.

Pinus Strobus.

"Austriaca.

sylvestris.

Tsuga Canadensis.

Larix laricina.

\section{Cryptogamia-Flower- lesw planta. FERNS.}

Pteris aquilina.

Asplenium Filix-fœmina. platyneuron.

Aspidium Noveboracense. acrostichoides. marginale.

Onoclea sensibilis.

Osmunda regalis. cinnamomea. ()

Weisia viridula.

Dicranella heteromalla.

Ceratodon purpureis.

Barbula unguieulata.

Catharinea undulata. i6 angustata. crispa.

Pogonatum brevicaule.

Polytrichum juniperinum. comnune.

Bryum argenteum. Inium cuspidatum.

$$
\text { atfine. }
$$

Physcomitrium pyriforme. Cylindrotnecium seductrix. Amblystegium serpens.

Eurhynchium Boseii.

Brachythecium plumosum. is

Riccia nutans. HEPATICS.

\section{BROOKLYN PARKS.}

Prospect Park contains 516 $\frac{1}{6}$ acres. In woodland, 110 acres; in lakes and watercourses, $7 \%$ acres ; in meadows, 70 acres ; in plantations, 2593 acres; in drives, $83 / 4$ miles; bridle roads, $3{ }_{2}^{2}$ niles: walks, $103 / 2$ miles.

Ocean Parkway is 5 1/2 miles long and 210 feet wide. Eastern Parkway is $21 / 2$ miles long and 210 feet wide.

\section{A rea of other Parks.}

Coney Island Concourse............ 0 0 acres Parade Ground . . ................. 40 Washington Park $\ldots \ldots \ldots \ldots \ldots \ldots \ldots$ so " Tompkins Park ................... City Park ........................ $73 / 2$

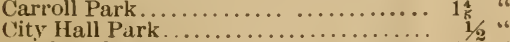
City Hall Park..........................

Total. $161 \frac{1}{2}$ "

The construction of Prospect Park began in June, 1866, and ceased in $18 \% 4$, being about twothirds finished. The land cost $\$ 3,919.3 \% 0.70$; construction cost $\$ 5,239,964.11$. Total, $\$ 9,159,334.31$.

\begin{tabular}{|c|c|c|c|c|}
\hline Month. & 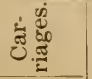 & 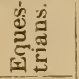 & 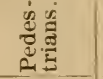 & $\begin{array}{l}\overrightarrow{\Phi ే} \\
\overrightarrow{0}\end{array}$ \\
\hline Nov & 415,458 & 4,617 & $109,3 \% 6$ & 529,454 \\
\hline lee: & $5,32,618$ & $8,13: 3$ & $12 \tau, 064$ & 667,815 \\
\hline JaIl.. & 547,826 & 5,559 & 119,467 & $6 \% 2,852$ \\
\hline $\mathrm{F}(\mathrm{l}) \ldots$ & 196,651 & 2,790 & 145,630 & 350,001 \\
\hline March & 571,500 & 6,645 & 173,146 & 751,291 \\
\hline April.. & $4 \$ 1.095$ & 5,717 & 171,401 & 624,213 \\
\hline Mav.. & 6413.817 & 7.837 & $309, \times 50$ & 964,504 \\
\hline June & 586,$31 ; 9$ & 12,922 & 646,320 & $1,216,111$ \\
\hline July .... & 480,548 & 4,222 & $\tau 58,08 i$ & $1,212,857$ \\
\hline August.. & $640,83: 3$ & $6,5: 34$ & 821,810 & $1,469,17$ \\
\hline Sept ... . & 538,911 & 4.743 & 3.59 .041 & 902,695 \\
\hline October.. & 509,016 & 2,936 & 193,073 & 705,025 \\
\hline Total. & $6,113,142$ & $\approx 2,655$ & $8,940,265$ & $10,126,065$ \\
\hline
\end{tabular}
Visito r\&, 1कर्?.
Total number visitors on Sundays, 2,364,138. Total number visitors on concert days, $90 \%, 8 \% 3$. Largest number in one day, June $23,11 \%, 523$.

\section{Saturday Concerts.}

Prospect Park, 15; first concert, June 8; last concert, September 28.

Washington Park, 16; first concert, June 8; last concert, September 28

\section{SUNDAY CONCERTS.}

Prospect Park, 13; first concert, July $\tau$; last concert, September 29 .

Two concerts for benefit of Johnstown Sufferers, Sunday. June 16 and Sunday, June 23.

Sports at Prospect Park.

Pienics, 390; base ball games, 1,295; foot ball games, 34; cricket gantes, 92; lacrosse ganles, 18

Number of skating lays, 3 ; first day's skating, February 15; last day's skating, February 25.

\section{Park Police Force.}

One captain, 7 sergeants, 62 patrolmen. Total force, 72 . Distributed as follows: Prospect Park, 6 sergeants, 49 patrolmen; Washington Park. 1 sergeant, 6 patrolmen; City Park, 2 patrol men; Tompkins Park, 2 patrolmen; Eastern Parkway, 1 patrolman; Ocean Parkway, patrolman; carroll Park, 1 patrolman.

\section{ACCIDENTS.}

Carriages and sleighs, $12 \%$; saddle loorses, 16 : bicycles and tricycles, 8 ; fell in lake, 6 ; fell in Park, 8; fell on ice, 1; taken sick in Park, 8; fracture of leg by falling from wall of Washington Park, 1: drowned on East side lands, 1: hurt on ball grounds, 1; struck with stone, 1. Total, $17 \times$. 
ร

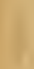

$=$

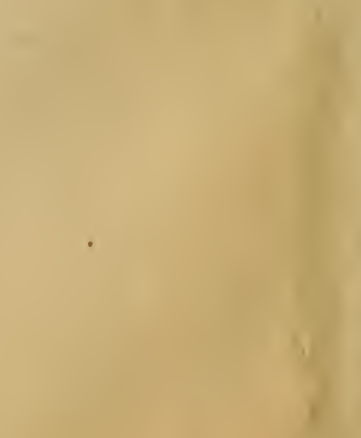

